\title{
Mort et disparition durant la dernière dictature militaire argentine (1976-1983) : les « disparus » et les soldats tombés lors de la Guerre des Malouines
}

Laura Marina Panizo, Docteure en Anthropologie sociale de l'Université de Buenos Aires. Enseignante-chercheuse à l'Instituto de Altos Estudios Sociales/ UNSAM.

Texte traduit de l'espagnol (Argentine) par Alice Verstraeten.

\section{Résumé}

Je propose de synthétiser dans ce travail les résultats de mes recherches sur la problématique de la mort violente et de l'absence de corps qui, dans le cadre de la dernière dictature militaire argentine, ne concerne pas seulement les victimes de la répression étatique mais concerne aussi les soldats tombés pendant la Guerre des Malouines. Deux groupes de parents se sont formés à partir de la mort et de la disparition d'êtres chers, puis ont élaboré des cadres d'interprétation pour donner du sens aux événements historiques survenus et pour surmonter les pertes.

\section{Le contexte}

Durant la dernière dictature militaire argentine (1976-1983), la société souffrit la perte violente de plus de 10.000 citoyens, victimes de la répression illégale et de la Guerre des Malouines. D'une part, le gouvernement fut responsable de la violation systématique des Droits Humains, par sa stratégie répressive fondée sur la détention clandestine, l'assassinat et la disparition physique de milliers de personnes. D'autre part, en 1982, le gouvernement militaire décida de récupérer les Iles Malouines, ce qui conduisit à un conflit armé entre l'Argentine et la Grande-Bretagne. Les Forces Armées Argentines se rendaient le 14 juin de la même année, après avoir perdu 649 soldats. 
Nous tenterons de synthétiser ici les résultats de nos recherches doctorales (Panizo, 2011), dont l'objectif était d'analyser comment les proches de disparus victimes de la répression et les proches de soldats tombés pendant la Guerre avaient affronté la problématique de la mort, en se penchant sur l'absence de corps et l'impossibilité de réaliser les rites de mort socialement établis.

Pour ce faire, nous avons travaillé avec des proches de ces disparus et de ces morts, appartenant à l'organisation « Familles de Disparus et Détenus pour Raisons Politiques » et à la «Commission des Familles de Soldats tombés pendant la Guerre des Malouines et des Iles de l'Altlantique Sud». Ces deux situations spécifiques ont produit une rupture dans les façons habituelles d'affronter la mort, et les proches ont dû, dans les deux cas, s'habituer à ces changements pour surmonter les pertes, réélaborant de manière créative les cadres conventionnels de compréhension de la mort.

Considérons la mort comme un processus social, survenant après la rupture des relations sociales qu'est le décès. La mort est alors un processus dans lequel nous nous investissons par le biais de différentes étapes de séparation et réintégration, dans lesquelles le groupe et la communauté jouent un rôle fondamental. Dans ce processus de transition, qui suppose des temps et des rituels spécifiques, et dont le corps est le principal symbole, la reconnaissance sociale et étatique est de la plus haute importance. Nous observerons, dans le cas de la Guerre des Malouines, malgré l'absence de corps, la présence de pratiques mortuaires ainsi que de pratiques étatiques de légitimation officielle du décès. A l'opposé, nous trouvons le cas des disparus, dans lequel il n'y a non seulement pas un mort - pas un corps - à qui rendre culte et prêter attention, mais il n'y a pas non plus d'espaces sociaux qui portent appui, contention ni attention aux personnes endeuillées.

Nous verrons également que nos interlocuteurs construisent leurs cadres symboliques d'interprétation à travers une sélection de symboles et une idéologie spécifique ${ }^{\mathrm{i}}$, qui orientent leurs manières de donner sens à la mort dans le contexte de la Guerre et de la disparition. Les représentations générées dans ces cadres interprétatifs répondent à des représentations, valeurs et pratiques structurées à niveau national. Ainsi observerons-nous, par exemple, que la figure du héros ou les distinctions nationales deviennent des outils culturels exprimant et mettant en scène diverses significations qui permettent aux proches de comprendre leur relation à leurs parents morts, mais aussi leur relation à l'histoire culturelle. 
Ainsi les cadres symboliques des groupes sont-ils des répertoires symboliques qui orientent les pratiques et les significations des familles, pour répondre à la problématique de la mort en accord avec leurs intérêts et pour symboliser un type de relation spécifique entre les familles et leurs proches. En ce sens, les rites de mort et les pratiques de la mort sont compris non seulement comme des instances d'adieux, de séparation et d'intégration des sujets sociaux (vivants comme morts), mais aussi comme des moyens expressifs de communication qui rendent compte du cadre social à travers lequel les individus donnent du sens au passé et au présent, et s'identifient à un groupe.

\section{Les disparus de la dernière dictature militaire argentine}

A partir de la disparition de personnes, différents organismes de défense des Droits Humains se sont formés en Argentine, réclamant à l'Etat mémoire, vérité et justice. Parmi ces différents organismes, j'ai travaillé avec celui des Familles de Disparus et Détenus pour Raisons Politiques, né officiellement, à Buenos Aires (capitale fédérale), en septembre 1976. Cette organisation, en plus de produire un discours ancré dans la dénonciation humanitaire, s'est caractérisée par 1) la revendication de la militance de leurs proches disparus ; 2) la réclamation de «l'apparition en vie» des disparus et de la libération des prisonniers politiques; et 3) la non-acceptation publique des morts jusqu'à ce qu'apparaissent corps et coupables. Puisque la méthodologie de la disparition de personnes se caractérisait, après l'assassinat, par le fait de ne pas annoncer la mort et de ne pas laisser apparaître de corps, les familles n'ont pu accéder à une connaissance claire du fait et des formes de la mort et ont été empêchées de réaliser quelque rituel conventionnel de mort que ce soit. De cette manière, la figure du disparu qui faisait, au départ, référence à une personne qui aurait été soustraite de la vie sociale, et dont on ignorerait où elle se trouve, était synonyme de recherche. Une fois que les familles prirent connaissance de l'existence des centres clandestins de détention et des tortures exercées sur les détenus/disparus, il y eut également le fantasme de retrouver des disparus désorientés, rendus amnésiques par exemple, et l'espoir de les retrouver alors en pleine rue. Avec le temps, après les témoignages des ex-détenus, le rapport Nunca más $(1984)^{\mathrm{ii}}$, le Procès des Juntes $(1985)^{\mathrm{iii}}$ et les identifications de l'EAAF $^{\mathrm{iv}}$, la recherche se transforma en recherche du corps et de la mémoire, la vérité et la justice. 
La revendication de la militance de leurs proches disparus, de la part d'une organisation qui, dès ses premiers pas, est engagée politiquement, est un facteur clé pour comprendre l'intention de maintenir le disparu comme sujet actif, comme partie prenante de la recherche de la vérité et de la lutte politique contre la dictature. Il faut souligner que l'une des caractéristiques essentielles de l'organisme est d'avoir reconnu, dès l'entrée, que ses proches disparus et détenus avaient, dans leur majorité, un lien étroit à la politique, raison pour laquelle, au moment de choisir le nom de l'organisation, fut ajouté le «pour raisons politiques », qui assumait cette réalité et en témoignait de la fierté. C'est pour cette raison que dès ses premières années, non seulement cet organisme lutta pour la recherche des disparus, mais se mit en lien avec d'autres secteurs sociaux dont ses militants considéraient qu'ils voyaient également leurs droits bafoués, maintenant ainsi la ligne idéologique de leurs proches absents. C'est dans la même logique que ces membres militent pour que les responsables des disparitions et assassinats soient poursuivis en justice, et pour que les disparitions ne deviennent pas des morts tant que la justice ne sera pas advenue.

Pour ceux qui n'eurent pas la possibilité de récupérer le corps, son absence et le manque de signes de mort, qui entravent sa reconnaissance sociale, peuvent rendre difficile la réalisation de rites de mort collectifs. Le mort, qui est en même temps un vivant, ou qui n'est ni mort ni vivant, ne parvient jamais à entrer dans le monde des morts et cela rend difficile, pour l'endeuillé, tant la réintégration adéquate à la vie sociale que le remplacement du lien brisé. Dès lors, les proches distinguent deux types opposés d'approche de la perte, celle qu'ils appellent l'approche «logique» ou «rationnelle», qui accepte la mort, et celle qu'ils appellent « irrationnelle » ou affective, celle qui vient « de l'intérieur », et où surgit l'espoir. Tant que ni le corps ni une information spécifique sur la mort n'apparaissent, ces deux types d'approche de la mort interagissent comme deux modalités possibles de la réalité. Cette ambiguïté existentielle, ajoutée au manque de pratiques mortuaires spécifiquement référées aux disparus en général, bloque le passage du sujet rituel de la vie à la mort. En ce sens, j'ai proposé de parler de mort ignorée (muerte desatendida), quand la mort devient un phénomène auquel on ne prête pas d'attention rituelle. Je parle de mort ignorée dans les cas où il n'y a pas de mort à qui rendre un culte, et où ne se produisent pas les pratiques rituelles apportant appui et contention aux endeuillés. Dans ce type de mort, les proches ne disposent pas d'espaces sociaux où, à travers une action collective, l'état de mort du disparu serait légitimé.

Dans les cas d'une mort ignorée, il ne se produit pas d'actions rituelles qui assigneraient, au sein de la structure sociale, aux uns le rôle de morts, aux autres celui 
d'endeuillé. Il s'agit donc d'une mort sans corps présent, aux aspects ambigus et paradoxaux. En ce sens, les disparus peuvent être considérés comme des êtres liminaux, qui sont sur le point d'être quelque chose, ou qui sont et ne sont pas à la fois ${ }^{\mathrm{v}}$. Ils sont de l'ordre de l'interstructurel, ils ne sont ni morts ni vivants, mais vivants et morts à la fois, ils ne sont ni ici ni là-bas et ils sont partout (Panizo, 2013 a).

Une pratique montre l'ambiguïté de la disparition: celle des avis publiés quotidiennement, depuis la fin des années 80, dans le journal Página 12 de Buenos Aires ${ }^{\mathrm{vi}}$. Le quotidien les publie gratuitement, et il s'agit de messages qui rappellent la date de naissance et d'enlèvement du disparu. Dans certains cas, on y détaille des éléments sur la disparition ou la mort, on y fait des réclamations et des dénonciations et, généralement, les textes sont accompagnés d'une photo du disparu en question. Il faut prendre en compte la variété des messages portés par ces souvenirs publiés, puisque nous en trouvons qui demandent des informations sur le destin de leur proche et vont jusqu'à donner une adresse où envoyer des informations ; nous en trouvons qui convoquent à des cérémonies et des messes ; d'autres qui s'adressent aux disparus; d'autres encore qui laissent des messages au public en général, l'informant de la disparition et du Terrorisme d'Etat; d'autres qui réclament mémoire, vérité et justice ; ou d'autres dont le but est de rappeler l'anniversaire de la disparition ou de la mort. Dans ces derniers cas, il est intéressant de souligner que ces souvenirs publiés marquent une claire différence entre les allusions aux assassinats, aux morts et les allusions aux disparitions. Quelques membres de l'organisme des Familles de disparus ont, par exemple, modifié le style des sollicitations après avoir récupéré le corps de leurs proches, par le biais de l'Equipe Argentine d'Anthropologie Légiste. Ainsi, après l'identification du corps, les sollicitations ne faisaient plus seulement allusion à un disparu, mais à un assassiné. Sans écarter le fait que pour certaines familles, ces souvenirs publiés puissent en venir à avoir une fonction mortuaire, nous pensons, en général, qu'ils marquent une continuité avec les pratiques habituelles des familles en matière de recherche de leurs enfants, de leurs corps, et avec le slogan mémoire, vérité et justice.

Une autre pratique qui exprime et légitime l'ambiguïté existentielle de la disparition est la «Loi 24.321, Disparition forcée de personnes » ${ }^{\text {vii }}$. La promulgation de cette loi est l'une des plus grandes victoires que s'attribue l'organisme, puisqu'elle apparait comme une victoire face à l'obligation, pour toute action légale du type déclaration de droits parentaux ou indemnisations, de déclarer mort le disparu. Le projet a été conduit par les associations des Familles de Disparus et Détenus pour raisons politiques, des Mères de la Place de Mai - 
Ligne Fondatrice et des Grands-Mères de la Place de Mai, et discuté par divers organismes de défense des droits humains avant d'être porté devant la Chambre des Députés. Cette nouvelle loi venait remplacer la «Loi d'absence avec présomption de décès » (Loi 14.394), avec un pouvoir rétroactif. Les effets civils des deux lois sont les mêmes, mais à l'inverse de la première, la dernière n'implique aucune reconnaissance de la mort par les juridictions civiles. Avec la nouvelle loi, l'on reconnaît la figure de l'absent pour disparition forcée, sans nécessité de déclarer la mort. Une nouvelle catégorie juridique s'institue alors, et une nouvelle forme d'administration de la mort est mise en évidence, dans le but de régulariser et réparer, depuis l'Etat de droit, les dommages causés par les gouvernements dictatoriaux ${ }^{\text {viii }}$.

Ainsi, si au départ le disparu se trouvait dans les interstices de la structure sociale, la loi citée ici réinscrit la figure de la disparition dans le registre du socialement établi et permis, elle la réinsère dans la structure, la rend visible. Le liminal (compris comme à la limite de la vie et de la mort) quitte alors sa marginalité structurelle, tout en maintenant un haut degré d'ambiguïté et de paradoxe. Depuis le social, si la mort n'est pas habilitée, l'état de liminalité est légalisé. Ainsi le terme de disparition fait-il référence à une condition d'ambiguïté structurelle où les sujets, soustraits à la réalité, sont sur le point d'être morts mais ne le sont pas tant que n'apparaissent ni les corps ni les coupables.

La liminalité, la disparition socialement reconnue et la mort ignorée permettent aux proches de disparus de résister politiquement à quelque pratique sociale que ce soit qui tenterait de donner leurs êtres chers pour morts et ce, jusqu'à la récupération du corps. A partir des exhumations réalisées par l'Equipe Argentine d'Anthropologie Légiste, la mort ignorée se convertit en une mort socialement reconnue; le phénomène du décès est socialement reconnu et la condition sociale de chaque personne se reconstruit.

\section{Disparition dans le cas de la Guerre des Malouines}

Bien que l'Etat dictatorial n'ait pas fait de recherches ni clairement informé qui que ce soit des modalités de la mort durant la Guerre des Malouines, les morts des soldats tombés dans ce conflit ont été comprises par ce pouvoir, à la différence de celles des disparus, comme « concrètes », « nationales » et « héroïques $»^{\mathrm{ix}}$. 
A l'image des organismes liés à la disparition, différentes organisations nongouvernementales - organisations civiles ou de vétérans - se formèrent à la fin de la Guerre des Malouines. La plus visible dans l'arène publique reste, de la fin de la guerre à nos jours, celle des «Familles de soldats tombés aux Malouines et dans les Iles de l'Atlantique Sud » (qui se constitue en commission, à Buenos Aires - Capitale fédérale, en 1982).

Contrairement aux associations de proches de disparus, cette commission ne se fonde pas sur des réclamations et des protestations dirigées vers l'Etat National, mais se propose d'honorer publiquement les êtres chers morts pendant la Guerre, de réaffirmer la souveraineté argentine sur les Malouines, de promouvoir toutes sortes d'activités qui réaffirmeraient le « concept d'argentinité » ou renforceraient « les valeurs culturelles, spirituelles et sociales qui caractérisent le peuple argentin ». Ainsi, dans le paysage symbolique de l'histoire argentine, parmi les symboles de l'unification de la diversité culturelle, les familles des Malouines se placent sous les signes de la patrie, de la religion et des ancêtres : elles revendiquent l'engagement sous le drapeau, se réapproprient l'image de la Vierge de Luján, et identifient leurs proches aux héros nationaux consacrés par 1'histoire officielle. Dans le paysage des représentations associées à l'histoire récente, cette fois-ci, dans le contexte de la dictature militaire, alors que le statut de victime et les dénonciations de violations des droits humains étaient associées aux disparitions de personnes, l'élaboration du sentiment de la mort des soldats s'inscrivit davantage dans le registre de l'unité nationale que dans celui de la dénonciation sociale.

A partir de l'objectif premier, pour les familles, d'honorer publiquement les héros, des activités se développent. Au fil de ces activités se créent rituels et espaces mortuaires où les endeuillés affrontent la mort «en face », dans des espaces d'attention rituelle. Ces activités finissent donc par fonctionner comme des espaces sociaux où les soldats tombés à la guerre ne sont plus seulement présentés comme des héros nationaux. Et c'est indispensable pour ces familles qui, comme celles des disparus, sont confrontées à l'absence de corps et à l'impossibilité de réaliser quelque rituel mortuaire conventionnel que ce soit.

Ce conflit s'est soldé par 469 victimes, et le cimetière de Darwin ne compte que 237 sépultures, réparties en 230 tombes individuelles et deux fosses communes : 1'une comptant 4 membres d'équipage d'un hélicoptère et l'autre comptant 3 membres d'équipage d'un Lear Jet abattu. Des 230 tombes, 123 possèdent des pierres tombales avec les mots « Soldat connu 
de Dieu seul » et 107 tombes, ainsi que les deux fosses communes, possèdent des pierres tombales où sont inscrits les noms et prénoms des personnes enterrées. Manquent, dans ce cimetière, les 323 victimes du naufrage du croiseur ARA General Belgrano, 2 membres d'équipage du Lear Jet enterrés sur l'Ile de Bourbon, différents marins perdus avec leurs navires, un certain nombre de pilotes abattus et quelques soldats enterrés sur l'Argentine continentale. Ainsi les familles manifestent-elles les difficultés qu'elles eurent à affronter la mort, alors que les pratiques rituelles habituelles étaient empêchées.

Pour contourner les obstacles et les ambiguïtés produites par l'absence de corps, il fut important pour les familles, non seulement de s'appuyer sur les recours de l'Etat - avec, par exemple, les termes de la Loi d'absence avec présomption de décès (Loi 14.394) -, mais également de s'appuyer sur l'histoire nationale. C'est pourquoi elles mettent l'accent sur la figure du héros plutôt que sur celle de victime, elles cherchent à abstraire cette guerre du Terrorisme d'Etat et proposent d'inclure les soldats tombés à la guerre au panthéon de référence des illustres citoyens de la Nation. Les héros des Malouines se distinguent alors des autres morts et se distinguent des disparus, dont on pense comprendre qu'ils ne sont pas morts en défendant un territoire national menacé par des forces étrangères. Dans l'idée que les soldats tombés ont donné leur sang pour le territoire de tous, pour une cause nationale, les familles veulent penser la Nation comme une communauté morale, qui dépasse les gouvernants. Elles utilisent pour cela une métaphore organique, avec l'association symbolique corps-nation, présente dans toutes les représentations des familles pour légitimer l'incorporation des soldats tombés aux Malouines dans le lignage des illustres citoyens de la Nation (Guber 2001 ; Lorenz 2006). Une fois admis que leurs êtres chers donnèrent leurs vies pour la Patrie, une fois ceux-ci consacrés par l'Etat argentin, à travers la Loi nationale 24.950, comme des héros nationaux ${ }^{\mathrm{x}}$, l'objectif principal de la commission des familles fut de préserver la mémoire de soldats et de promouvoir une attitude sociale d'hommages publics, comme cela se faisait pour les morts illustres qui les avaient précédés.

La catégorie du héros renvoie, dans ces cas-là, à des vies tragiquement interrompues pour une cause nationale qui donc, méritent une reconnaissance sociale particulière. Dans ce cas précis, l'acte héroïque consiste, pour les proches également, en un sacrifice pour la patrie. Nombreux sont les proches qui soulignent alors l'existence de valeurs communes entre les soldats tombés aux Malouines et San Martin et Belgrano, pères fondateurs de la patrie : les valeurs de la responsabilité sociale. En activant les symboles offerts par l'histoire nationale, les familles construisent un nouveau panthéon de héros, et une mémoire de la guerre qui exige 
de ne «pas oublier » les actes héroïques des soldats. Pour que la cause des Malouines de soit pas dévalorisée par les condamnables conduites des militaires face aux disparus, beaucoup de familles tentent de séparer la guerre du fait qu'elle ait été conduite par une dictature militaire responsable de la disparition de personnes et de l'appropriation illégale de bébés nés en détention. On valorise le rôle du soldat tombé en tant que citoyen, pour défendre un territoire qui, selon l'expression des familles « appartient à tous » et ce, sans introduire les différences politiques du moment. De cette manière, le soldat tombé aux Malouines unit les Argentins face à un ennemi extérieur.

Ce qui ressort de la catégorie du héros national est la dimension sacrificielle. C'est ainsi que nous pouvons interpréter les récits de leurs proches, et surtout de leurs mères qui, en soulignant leurs réticences à voir partir leurs enfants à la guerre, soulignent du même coup la décision et la volonté propres de leurs enfants. C'est ce qui fait d'eux des êtres sacrificiels : ce sont eux-mêmes qui offrent leurs vies pour consacrer la patrie.

Si dans l'arène publique, on laisse entendre que l'objectif principal des familles est d'obtenir que la société dans son ensemble confère aux soldats tombés la gloire et le statut de héros patriotiques, nous interprétons cette volonté de rapprochement avec l'institution comme la mise en place d'une relation spécifique aux morts qui permette de soulager les douleurs et les culpabilités dues à ce contexte de mort violente à la guerre, d'absence de corps, d'impossible rituel conventionnel, de manque d'un adieu adéquat. En ce sens, ce mode de compréhension basé sur la tradition catholique argentine et la mythologie héroïque nationale propose également des formes alternatives de relation entre les endeuillés et leurs morts compris, comme nous l'avons écrit, comme des martyrs volontaires-. Parmi ces formes alternatives de communication avec les morts, nous trouvons ce que nous avons appelé « autels domestiques », qui se construisent autour d'un objet principal à qui l'on rend le culte : la photographie du mort. Elle est entourée d'autres objets symboliques, comme l'image de la Vierge de Luján, des rosaires, des croix, des diplômes, des objets de commémoration, ou des objets que les familles ont ramené des leurs différents voyages sur les îles, comme du sable, des coquillages, des petits morceaux de bois, etc. Dans ces espaces domestiques, transformés en lieux sacrés où elles les vénèrent «comme des Saints », les prient et les sollicitent, les familles sont étroitement connectées à leurs proches. Les ressources culturelles du catholicisme et la religiosité populaire y deviennent évidentes. 
C'est donc la mort à la guerre, le manque de corps et l'idée de sacrifice volontaire qui confèrent aux morts le statut de saintetés et permet aux familles d 'établir une sorte de relation réciproque avec eux, de les maintenir au rang d'agents actifs de leurs vies quotidiennes. On confère alors aux morts une part de vitalité et l'on a avec eux des expériences extraordinaires qui peuvent se comprendre comme des signaux, ou comme des formes de communication, à travers notamment les prières et les demandes d'aide. Dans les relations que les familles construisent avec leurs proches, l'on ressent leur présence permanente et leur soutien dans les moments difficiles de la vie.

Outre les autels domestiques, les familles trouvent d'autres façons d'entrer en relation avec leurs morts, et ce à travers divers rituels où la Vierge de Luján joue un rôle fondamental : elle devient un symbole dominant de l'action rituelle en même temps qu'elle condense des références à la guerre, à la nation, au patriotisme, à la protection et à la maternité. La Commission des familles utilise alors une icône de l'identité nationale catholique, sujette à une forte dévotion populaire depuis son arrivée en Argentine et nombre de miracles qui lui ont été attribués. C'est une façon de récupérer le capital symbolique des héros, capital entamé par l'action infamante de l'Etat et de la société.

En plus d'avoir participé à la majorité des activités de la Commission, la Vierge a presque parcouru l'ensemble des provinces du pays au fil de ses pèlerinages. Les pèlerinages ont permis d'amener aux différentes parties du territoire, à travers la Vierge, le souvenir de la Guerre. La Vierge reçut alors différentes prières et offrandes de la part de tous les argentins et ce, jusqu'au 10 octobre 2009, date à laquelle elle fut amenée au cimetière de Darwin pour y rester aux côtés des tombes et du monument aux soldats morts de l'Ile Soledad. Ce sont donc des argentins de toutes les provinces qui ont fait des offrandes à la Vierge avant son départ pour les Iles, lui demandant -entre autres choses - de veiller sur les héros. La manifestation à la fois symbolique et matérielle d'offrandes et prières des fidèles à la Vierge avait, selon les angles d'approche, différents objectifs simultanés. D'un côté, son culte renvoyait au culte des morts. La Vierge, qui incarne alors le corps symbolique de la nation, apporte avec elle le souvenir de la guerre et de ses héros aux différents villages argentins. D'un autre côté, ce culte fut compris comme une récompense pour la protection spirituelle qu'elle avait apportée aux héros sur les Iles. Mais là aussi, la Vierge est un lien entre les argentins de divers horizons géographiques et le royaume des morts, à travers les offrandes et les prières. Enfin, à travers l'image de la Vierge, mère protectrice, les mères des Malouines étendent leurs liens et leurs soins maternels, interrompus par la guerre et entravés dans la mort par l'absence de corps. 
Ainsi la Vierge se convertit-elle en messagère, canal de communication entre différentes communautés et entre les vivants et les morts. A travers cette fonction unificatrice du héros comme de la Vierge, c'est l'identité nationale qui est valorisée au détriment des autres identités, c'est la religion catholique qui est valorisée au détriment des autres religions. A travers la métonymie Vierge/Nation, à travers son pouvoir surnaturel de médiation entre les vivants et les morts, les familles confient à la Vierge non seulement la garde de leurs enfants, mais aussi celle des Iles, en la plaçant au cimetière de Darwin. Nous y reviendrons.

L’image de la Vierge de Luján a également joué un rôle fondamental dans ces autres rituels de mort que sont les messes du 2 avril. Le 28 mars 1983, le gouvernement dictatorial a, à travers la loi 22769, déclaré le 2 avril «Jour des îles Malouines, des îles de la Géorgie du Sud et des îles Sandwich du Sud» ${ }^{x i}$. Les familles ont donc choisi cette date pour réaliser, une fois par an, une messe en l'honneur des soldats tombés à la guerre, et ce dans la cathédrale métropolitaine de Buenos Aires qui, par ailleurs, abrite depuis 1882 les restes du Général San Martin, le Père de la Patrie. Lors de ces cérémonies, les familles, en guise de marche mortuaire, offrent des fleurs aux soldats tombés (649 boutons de roses rouges qui représentent chacun d'entre eux). Ces messes portent à la fois les caractéristiques d'un rituel mortuaire et celles d'une manifestation patriotique/ militaire. Car à ces offrandes florales et à la minute de silence dédiée aux morts se mêlent les présences de militaires et de la Vierge, ainsi que divers symboles patriotes, mettant publiquement en scène une façon particulière de comprendre la mort et mettant en évidence les liens de la Commission avec l'Eglise, l'Armée et l'Histoire nationale.

Si des cénotaphes et des monuments en l'honneur des combattants tombés aux Malouines ont bien été érigés dans divers villes argentines, le Monument (cénotaphe ${ }^{\mathrm{xii}}$ ) du cimetière de Darwin a la particularité, alors qu'il est promu et géré par la Commission, de se situer certes là où reposent les corps, mais également sur un lieu encore disputé, un lieu qui a motivé la guerre et dont le retour dans le giron national est toujours attendu. Le cénotaphe et le cimetière de Darwin constituent donc un fait inédit dans l'histoire des affrontements belliqueux, en ce qu'ils sont construits par les vaincus, et administrés par les vainqueurs. Par là-même, la Commission considère ces constructions comme l'une de ses plus grandes victoires : elles offrent un lieu symbolique aux morts, à l'endroit même où ils ont laissé la vie et perdu leurs corps. 
La Commission, soutenue par l'Etat, a pu concrétiser l'inauguration du monument en organisant deux voyages, les 3 et 10 octobre 2009, convoquant l'ensemble des familles. Comme dans une sorte de rituel mortuaire, l'inauguration officielle du cimetière et l'intronisation de la Vierge ont agi pour de nombreuses familles comme une clôture du processus rituel à travers lequel les endeuillés se séparent définitivement de leurs morts. Reprenant Arnold Van Gennep et son analyse des rites de passages, Pierre Bourdieu propose d'appeler ces de type de rites, «rites de consécration» ou «de légitimation», soulignant qu'ils séparent ceux qui participent à ces rituels de ceux qui n'y participent pas. La fonction essentielle du rite, affirme l'auteur, est «d'instituer une différence durable entre ceux qui sont concernés par le rite et ceux qui ne le sont pas »(1992, p.113). En comprenant le rite comme une consécration, qui constitue «solennellement [...] de manière licite et extraordinaire, une transgression des limites constitutives de l'ordre social », l'auteur nous amène à considérer que l'important n'est pas d'orienter l'attention vers le rituel de passage, mais plutôt vers la ligne de démarcation qu'il trace (ibid). L'interprétation qui en découle ici est que, pour que le sacrifice ne soit pas vain aux yeux des familles, alors même que la guerre est perdue, il est fondamental que l'échange contractuel que suppose toute idée de sacrifice ait bien lieu : en l'occurrence, que les corps qui reposent au cimetière permettent une certaine souveraineté sur les Iles. A partir de la guerre, les Iles ont commencé à prendre une place particulière dans la cartographie symbolique des familles, et cette transformation s'observe à travers le langage métaphorique des familles, qui situent la Vierge, le corps des héros et la terre pour laquelle ils ont combattu dans le même champ sémantique. Ainsi, à travers l'idée de sacrifice volontaire et à travers la métaphore organique qui associe le corps et le «sang versé » à la terre, les morts sont compris comme métonymie de la terre. Cette opération symbolique permet aux familles de comprendre leurs morts comme des ressources nationales, moteurs de la souveraineté. En ce sens, l'inauguration officielle du monument peut être comprise comme la clôture d'un rituel sacrificiel, dans lequel l'objet sacrifié se verrait officiellement consacré héros sacré et protecteur de la Patrie. C'est alors que la métaphore entendue dans la bouche d'un proche de soldat, qui voyait les corps comme des «drapeaux argentins appelant à notre reconnaissance », devient significative. Les corps deviennent des symboles condensant les projets politiques des familles, qui ne comprennent pas leurs morts comme des morts banals, sinon comme des héros nationaux qui, selon leurs termes, sont «passés dans la grande histoire» et doivent «continuer à monter la garde de l'Argentine ». 
Selon Van Gennep (2008) lorsque les rites mortuaires sont respectés, la dernière étape de ce qui est alors un rite de passage voit l'intégration des morts dans leur monde et la réintégration des vivants dans le leur, à travers la séparation définitive des participants au rituel (les morts et leurs proches). Il s'agira plutôt, dans le cas présent, d'une distinction entre sacralisés et non sacralisés. Le mort ne passe en effet pas au monde commun des morts, mais plutôt au monde des « héros morts » au même titre que d'éminents ancêtres. Cependant, ce qui distingue ces soldats tombés des autres héros, c'est la localisation spécifique de leurs corps, représentant symboliquement la souveraineté argentine sur les Iles. C'est de cette manière seulement que peut se produire la séparation définitive des endeuillés et de leurs morts : en les intégrant à un espace géographique symboliquement dense, pour les consacrer «morts spéciaux », par l'intermédiaire sacré d'un symbole catholique de l'argentinité par excellence.

Le cimetière délimite le profane et le sacré, en même temps qu'il unit, nous l'avons $\mathrm{vu}$, les Iles et le reste du territoire argentin, à travers les morts et la Vierge. Suivant Bourdieu, l'effet le plus important du rite - dans ce cas précis, l'apogée d'un rite sacrificiel - est qu'il consacre la différence : le héros est institué être patriotique et sacré, en même temps que les autres mortels sont désignés comme «ne pouvant prétendre à ce type de légitimation rituelle » (1992, p.115). Le pouvoir symbolique de ces corps au cimetière de Darwin, alors même qu'ils ne sont pas tous identifiés, ne s'exerce donc pas seulement dans le registre de la souveraineté sur un territoire disputé, mais joue également dans la consécration de sujets rituels comme des êtres prédestinés à lutter et à mourir pour la cause.

\section{Derniers mots}

Nous observons, au fil de ce travail, que les morts des disparus et des soldats tombés à la guerre s'inscrivent dans des cadres symboliques qui orientent les proches vers des manières de donner du sens à la mort d'êtres chers, en les investissant comme sujets historiquement signifiants. Mais les cadres interprétatifs des deux groupes, bien qu'ils partagent un même contexte historico-social, sont différents. Les familles se sont approprié des configurations culturelles de façon distincte, selon leurs expériences propres et leurs intérêts en tant que groupes. La mort avec absence de corps a donc provoqué, dans les contextes sociaux de la 
dictature, des approches originales de la perte, en fonction du sens donné à ces morts particulières et de la possibilité de réinterpréter les représentations sociales.

Nous l'observons, parmi ces représentations se trouvent celles qu'a organisé l'Etat, avec 1) la promulgation de lois ; 2) les initiatives politiques liées aux droits humains, comme la formation de la Commission Nationale sur les Disparitions de Personnes (CONADEP) et l'élaboration de son rapport Nunca más; ou 3) sa participation et son appui financier à des activités spécifiques comme la construction du Monument aux victimes du terrorisme d'Etat à Buenos Aires ou la construction du Monument aux soldats tombés durant la guerre des Malouines au cimetière de Darwin.

Mais les familles ont joué un rôle actif dans la conformation des représentations et des catégories sociales. Les décisions de l'Etat quant aux normes et aux lois, quant à la manipulation des corps et à la dénomination des morts, n'instaurent pas seulement des catégories sociales du type disparus en action, morts au combat, absent pour disparition forcée, héros de guerre... Elles instaurent également un «fond social » d'interprétation des morts.

L'analyse comparative des ces cas nous a permis de comprendre que la confrontation avec la mort est possible, malgré l'absence de corps, si l'on peut compter en première instance sur une reconnaissance officielle/ étatique des morts. D'autre part, l'existence d'un cadre social qui permette la pleine réalisation de pratiques mortuaires est capitale.

\section{Bibliographie}

BOURDIEU, P. (1993). "Los ritos como actos de institución”, dans En Honor y Gracia, J. PITT-RIVERS et J.G PERISTIANY (eds.) Madrid, Alianza Editorial.

CONADEP (1984). Informe Nunca más, Buenos Aires, Editorial UBA.

GEERTZ, C. (1997). La interpretación de las culturas, Barcelona, Gedisa.

\section{GUBER, R.}

- (2001). ¿Por qué Malvinas? De la causa nacional a la guerra absurda, Buenos Aires, Fondo de Cultura Económica. 
- (2004). De chicos a veteranos. Memorias argentinas de la guerra de Malvinas, Buenos Aires, ed. Antropofagia.

LORENZ, F. (2006). Las guerras por Malvinas, Buenos Aires, Editorial Edhasa.

PANIZO, L. (2011).

TURNER, V. (1997). La selva de los símbolos, Madrid, Siglo XXI.

VAN GENNEP, A. (2008). Los ritos de paso, Madrid, Alianza Editorial.

\footnotetext{
' Je m'appuie sur C. Geertz pour comprendre l'idéologie comme formulation symbolique de signification, qui surgit comme réponse à des états de tension pour «donner du sens à des situations sociales incompréhensibles" (1997:192).

ii Le Nunca más fut un rapport réalisé par la Commission Nationale sur les Disparitions de Personnes (CONADEP). La commission, instituée en 1983 sous le gouvernement démocratique de Raúl Alfonsín, avait pour objectif de recevoir des informations et des dénonciations de disparitions, d'enlèvements et de tortures subies pendant le régime dictatorial.

iii Les procès des militaires responsables des crimes commis continuent à l'heure actuelle.

iv L'Equipe Argentine d'Anthropologie Légiste (EAAF) est une organisation non gouvernementale, à but non lucratif, formée en 1984 dans le but d'enquêter sur les cas de personnes disparues en Argentine durant la dernière dictature militaire. C'est ainsi que des anthropologues légistes, des anthropologues sociaux et des archéologues, depuis leur champ disciplinaire de la bio-anthropologie, utilisent techniques et méthodes archéologiques pour récupérer les corps inhumés en terre. Pour approfondir les différentes étapes de recherche nécessaires aux identifications, consulter leur site : http://www.eaaf.org/

$\checkmark$ Victor Turner, dans son analyse des rites de passage, développe les propriétés socioculturelles de la phase de transition, qu'il appelle liminale (1997). Les sujets rituels, dans la phase liminale, entrent dans un état ambigu et paradoxal. Les êtres liminaux n'ont rien : ni statut, ni rang, ni parenté. Ils appartiennent au « règne de la possibilité pure " (ibid : 107).

vi Página 12 est un journal du matin qui, dès sa création (1987), a un profil idéologique de centre gauche, impliqué aux côtés de la démocratie et des organismes de défense des droits humains (Van Dembroucke, 2004).

vii Loi votée le 11 mai 1994 et promulguée le 8 juin de la même année.

viii Fondements du projet de loi, "Déclaration d'absence pour disparition forcée ", texte présenté devant la Chambre des Députés de la Nation par le député Claudio Mendoza, le 7 décembre 1993, p.3936.

ix D'un autre côté, une fois terminé le conflit, les vétérans de la Guerre des Malouines furent compris comme des victimes de la dictature au même titre que les disparus. Les dénonciations des mauvais traitements infligés aux soldats par leurs supérieurs, ajoutées à leurs conditions de vie extrêmement dures, conduisirent les remises en cause des militaires à se rapprocher des questions de droits humains. (NE PAS METTRE EN NOTES MAIS EN TEXte PLEIN ?)

${ }^{x}$ La loi $n^{\circ} 24.950$, promulguée le 3 avril 1998, dispose que les combattants argentins décédés en défendant la souveraineté nationale dans les lles de l'Atlantique Sud en 1982 sont des " héros nationaux ».

xi Pour approfondir la question des enjeux de l'organisation de cérémonies officielles autour du 2 avril, en1983, consulter Guber, 2004.

xii Ce cénotaphe a été offert à la Commission par l'homme d'affaires Eduardo Eurnekian.
} 